Check for updates

Cite this: RSC Adv., 2019, 9, 40184

\title{
A facile fabrication of sepiolite mineral nanofibers with excellent adsorption performance for $\mathrm{Cd}^{2+}$ ions
}

\author{
Fei Wang, (D) *ab Ming Hao, ${ }^{\text {ab }}$ Jinsheng Liang, ${ }^{\star a b}$ Peizhang Gao, ${ }^{\text {ab }}$ Maomao Zhu, ${ }^{\text {ab }}$ \\ Baizeng Fang, (D) Hui Zhang ${ }^{\mathrm{ab}}$ and Zengyao Shang ${ }^{\mathrm{ab}}$
}

\begin{abstract}
In this work, sepiolite mineral nanofibers are facilely prepared by a microwave-hydrogen peroxide method, and the bulk densities of the samples are adopted to evaluate the defibering effect. The samples are systematically characterized through X-ray diffraction, scanning electron microscopy, specific surface area measurement and zeta potential determination, and the adsorptive performance for heavy metal ions in aqueous solution is studied using cadmium ions as the representative. It is found that the specific surface area and cumulative pore volume increase respectively up to $109.21 \mathrm{~m}^{2} \mathrm{~g}^{-1}$ and $0.234 \mathrm{~cm}^{3} \mathrm{~g}^{-1}$ under the microwave power of $400 \mathrm{~W}$, while the zeta potential reaches a maximum when the $\mathrm{pH}$ is 5.0 . The adsorption efficiency of sepiolite mineral nanofibers for cadmium ions can reach $68.6 \%$ as the optimal value. The as-fabricated sepiolite nanofibers can be regarded as a low-cost and environmentally friendly material which is a promising candidate for heavy metal ion removal from industrial wastewater.
\end{abstract}

Received 26th September 2019 Accepted 22nd November 2019

DOI: 10.1039/c9ra07836c

rsc.li/rsc-advances rheological, and catalytic properties due to the unique internal pore structure. ${ }^{12-14}$ The application of sepiolite mineral nanofibers has received extensive attention in environmental management, especially in the field of heavy metal wastewater treatments. ${ }^{15-18}$ Sepiolite mineral nanofibers have good adsorptive and ion exchange performance, which could be utilized to remove toxic pollutants, opposite charge ions, and complexes. However, natural sepiolite usually occurs as aggregates bundles in nature due to the special minerogenetic condition, ${ }^{19-21}$ which restricts considerably the adsorption capacity. Therefore, it is necessary to disperse sepiolite aggregates into individual fibers by micro-regulation of the structure. At present, the commonly used methods for preparing sepiolite mineral nanofibers are the hydrothermal, chemical lysis, and mechanical pulverization methods. ${ }^{22}$ The hydrothermal method has the disadvantages of small preparation amount and long cycle, and thus is unsuitable for mass production. Chemical lysis method releases the sepiolite fiber diameter only to submicron scale, and the mechanical pulverization method seriously reduces the ratio of the fiber length to diameter, and damages the sepiolite fiber structure as well. In recent years, microwave method has demonstrated some advantages over conventional heating method, mainly including short processing time, high efficiency and easy control, and is widely used in the process of building material structure. Microwave treatment studies on coal, ${ }^{23,24}$ carbon nanotubes,${ }^{25}$ graphite, ${ }^{26}$ sepiolite, ${ }^{27}$ nanoparticles, ${ }^{28-30}$ and vermiculite have been extensively studied. ${ }^{31,32}$ However, there is very few information about the removal of the inclusions in the mineral fiber pore micro-area 
and the regulation of the micro-structure of the inner pore of the fibers by microwave chemical method.

The objective of this work is to prepare sepiolite mineral nanofibers with good adsorption performance by the microwave-hydrogen peroxide method. The effects of reaction time and initial $\mathrm{pH}$ on the adsorption of cadmium ions were also explored. Besides, the adsorption mechanism was studied to establish a theoretical foundation for the research on the purification of heavy metal ions based on mineral nanofibers adsorbents.

\section{Experimental}

The raw sepiolite samples used in this study were acquired from Henan province of China, and the reagents including hydrochloric acid, sodium hydroxide, hydrogen peroxide, cadmium nitrate, nitric acid and ethanol were provided by the KW Reagent Incorporation. $500 \mathrm{~g}$ of the natural sepiolite sample (SEP) was added into $3112 \mathrm{~mL}$ of deionized water and $300 \mathrm{~mL}$ of hydrochloric acid solution (36 wt\%), and the mixture was stirred at a speed of $1500 \mathrm{rpm}$ for $1 \mathrm{~h}$ by a high-speed dispersing machine. After that, the slurry was filtered and washed several times with deionized water until the $\mathrm{pH}$ value was about 7 , and then the collected cake was dried at $80{ }^{\circ} \mathrm{C}$. Next, the dried cake was crushed and ground into powders using the 20 mesh sieve. The acid-purified sepiolite sample (A-SEP) was further mixed with $30 \%$ hydrogen peroxide at a solid-liquid ratio of 1 $\mathrm{g}: 20 \mathrm{~mL}$ and stirred for $3 \mathrm{~h}$. Later, the mixture solution was placed into a microwave vacuum tube furnace to prepare microwave chemical modified sepiolite (MH-SEP) under various microwave conditions.

A BRUKER D8-Focus X-ray diffractometer (XRD) was used to analyze crystallinity degree of the as-prepared materials. A XL30 scanning electron microscopy (SEM) was used to study morphological features. An Autosorb iQ type physical and chemical adsorption analyzer was employed to determine specific surface area, pore volume and pore size. A JS94H micro electrophoresis was used to examine the zeta potential. The bulk density of the samples was determined by measuring the mass of a fixed volume. A fixed volume glass cylinder was filled with the samples without vibration in accordance with the China Standard Test Methods (GB/T 16913.3-1997). The bulk density is calculated according to eqn (1):

$$
\rho_{\mathrm{p}}=\frac{m_{\mathrm{t}}-m_{\mathrm{v}}}{V_{\mathrm{v}}}
$$

where $\rho_{\mathrm{p}}$ is the bulk density of the sepiolite, $m_{\mathrm{t}}$ is the total mass of the sepiolite and the capacity cylinder, $m_{\mathrm{v}}$ is the mass of the capacity cylinder, and $V_{\mathrm{v}}$ is the volume of the sepiolite in the capacity cylinder.

A stock solution of $1000 \mathrm{mg} \mathrm{L}^{-1}$ of cadmium ion was prepared using analytical reagent grade cadmium nitrate dissolved in deionized water, and the above solution was diluted accordingly to give the appropriate concentrations. $100 \mathrm{~mL}$ of solution containing an appropriate amount of MH-SEP and with the initial concentration of $100 \mathrm{mg} \mathrm{L}^{-1}$ of heavy metal cadmium ions was added to a $150 \mathrm{~mL}$ conical flask, and was shaken for a certain period of time with THZ-82 gas bath temperature shaker at room temperature. After centrifugation, the concentration of cadmium ions in the filtrate was determined via a Thermo M6 atomic absorption spectrophotometer. Specifically, the initial $\mathrm{pH}$ value of the solution was adjusted to 2.0-7.0 with $0.1 \mathrm{~mol} \mathrm{~L}^{-1}$ hydrochloric acid or sodium hydroxide solution, and the shaking time was set as 5-180 min. The adsorption capacity and adsorption efficiency were calculated according to eqn (2) and (3) respectively:

$$
\begin{gathered}
q=\frac{\left(C_{0}-C_{\mathrm{e}}\right) V}{m} \\
R(\%)=\frac{C_{0}-C_{\mathrm{e}}}{C_{0}} \times 100
\end{gathered}
$$

where $V$ is the volume of the solution $(\mathrm{mL}), C_{0}$ and $C_{\mathrm{e}}$ are the initial and equilibrium concentration of the cadmium ions ( $\mathrm{mg}$ $\mathrm{L}^{-1}$ ), respectively, and $m$ is the mass of the MH-SEP (mg).

\section{Results and discussion}

Fig. 1 shows the variation of bulk density of sepiolite under different microwave powers. It can be seen that the bulk density decreases first and then increases with the increase of microwave power, and the bulk density of nanofibers reaches the lowest value when the microwave power is $400 \mathrm{~W}$. For the powders, smaller particle size would lead to lower bulk density, which is helpful for the improvement of adsorption capacity. ${ }^{33}$ In the microwave power range from 100 to $200 \mathrm{~W}$, sepiolite begins to absorb the microwave, and the bubbles produced are overflowing inside the sepiolite fibers with the increase of temperature, which brings about some gaps and increases the specific surface area of the sepiolite fibers. When the microwave power is in the range from 200 to $400 \mathrm{~W}$, the temperature rise of the system is accelerated, and the reaction rate reaches the maximum. However, the excessive temperature will destroy the optimal structure of sepiolite fibers when the microwave power

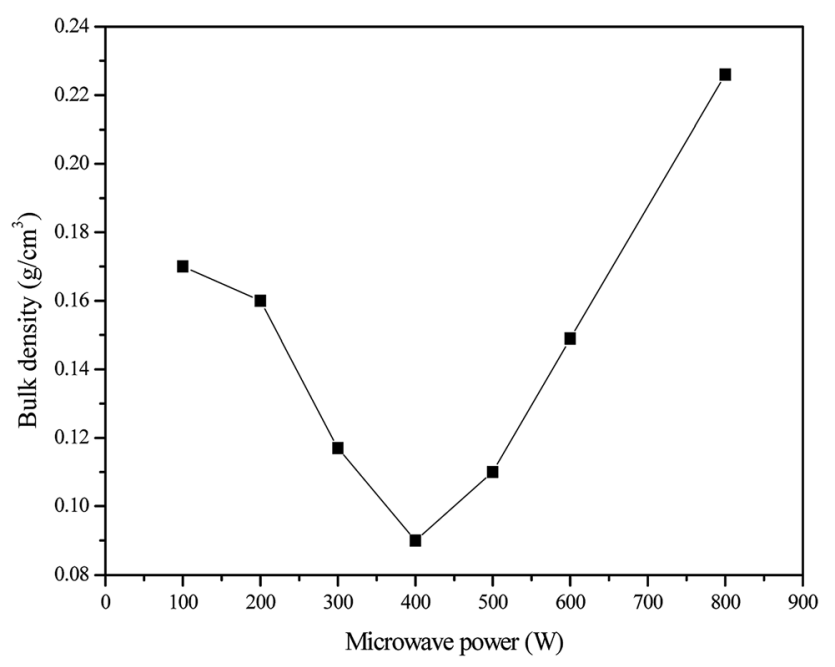

Fig. 1 Effects of microwave power on bulk density of the sepiolite samples. 
is continuously increased to $800 \mathrm{~W}$. Therefore, $400 \mathrm{~W}$ is selected as the optimal microwave power for the sepiolite nanofibers preparation.

Fig. 2 shows the XRD pattern of the various sepiolite samples in the preparation process. The characteristic diffraction peak of sepiolite has no obvious change before and after the treatments, indicating that the basic structure of sepiolite has not been destroyed. The as-received sepiolite (SEP) contains a lot of impurities such as calcite and dolomite, and their characteristic peaks disappear after the acid treatment. The characteristic diffraction peak of MH-SEP is widened after the microwavehydrogen peroxide treatment, which may be attributed to the lattice distortion and low degree of fiber aggregation. At the same time, (110) and (080) diffraction peaks of MH-SEP become significantly stronger, which indicates that the impurity content of the sepiolite is reduced and the purity is considerably enhanced.

Fig. 3 shows the SEM images of A-SEP and MH-SEP. As shown in Fig. 3(a), some fiber bundles have changed into individual fibers after the acid treatment, most of the A-SEP fibers are still bundled, long and thick. In addition, the fibers of the A-SEP have a sub-microscale diameter. In Fig. 3(b), the fiber bundle-shaped sepiolite disappears and the degree of fiber aggregation is obviously reduced after the microwave-hydrogen peroxide treatment, and the MH-SEP is mainly composed of the staggered loose sepiolite fibers.

Fig. 4 shows the cumulative pore volume and specific surface area of the various sepiolite samples. The cumulative pore volume and specific surface area of the MH-SEP are $109.21 \mathrm{~m}^{2}$ $\mathrm{g}^{-1}$ and $0.234 \mathrm{~cm}^{3} \mathrm{~g}^{-1}$, respectively, corresponding to 1.61 and 2.57 times of the SEP, respectively, indicating that the adsorption capacity of the MH-SEP can be expected be significantly higher than the unmodified one (i.e., SEP). The higher pore volume and specific surface area could be attributed to the fact that the hydrogen peroxide adsorbed by the sepiolite fiber channels rapidly decomposes and releases a large quantity of oxygen under the microwave heating, which leads to the

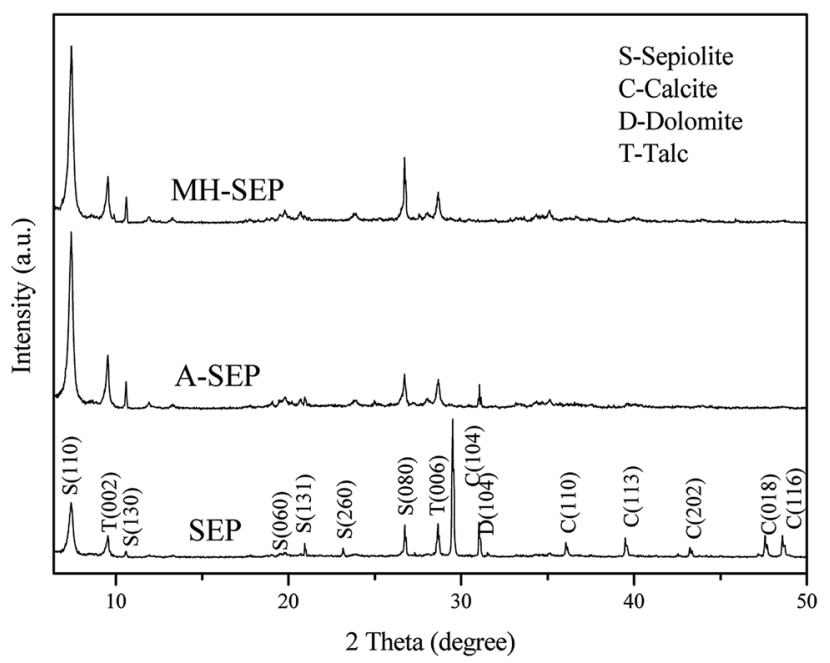

Fig. 2 XRD patterns of sepiolite samples. widening of sepiolite fiber channels in local high temperature and high pressure.

Fig. 5 shows the zeta potential of the various sepiolite samples in $\mathrm{pH}$ range of 2.0-7.0. The potentials of the three sepiolite samples decrease dramatically and then increase gradually, and the absolute value of the negative potential is the largest at $\mathrm{pH}$ 5.0. The surface is positively charged at $\mathrm{pH}$ values lower than 2.5, and the concentration of $\mathrm{H}^{+}$ions in the solution is higher than that of the $\mathrm{H}^{+}$ions adsorbed on the sepiolite, which leads to further adsorption of $\mathrm{H}^{+}$ions on the sepiolite surface. ${ }^{34}$ The potential shows a downward trend when $\mathrm{pH}$ is increased from 2.5 to 5.0, which is mainly due to the adsorption of $\mathrm{OH}^{-}$ions onto the positive charge center of the sepiolite or the deprotonation of the surface hydroxyl groups. ${ }^{35,36}$ The potential shows an increasing trend in $\mathrm{pH}$ range of 5.0 to 7.0, which can be attributed to the fact that dissolution rate of the equilibrium cations in the minerals decreases under weak acid conditions, and the mineral electronegativity starts to decrease. The absolute value of the zeta potential of MH-SEP increased significantly at the same $\mathrm{pH}$, which was much higher than that of the SEP. The above analysis reveals that surface negative charge increases after the sepiolite structure is optimized, which causes the positively charged cadmium ions to be more easily adsorbed through electrostatic interaction. Therefore the adsorption capacity could be enhanced.

Fig. 6 shows the effect of the reaction time on the adsorption of cadmium ions by MH-SEP at an initial $\mathrm{pH}$ of 5.0. It is evident that the adsorption capacity of cadmium ions by the MH-SEP increases rapidly at the beginning of the reaction, and then gradually after $30 \mathrm{~min}$ till the adsorption and desorption processes reach the equilibrium state. The adsorption process is the fastest at the beginning of $5 \mathrm{~min}$, and the adsorption efficiency is also increased along with the prolonging of reaction time up to $30 \mathrm{~min}$. The adsorption efficiency value at $30 \mathrm{~min}$ is a little smaller than that at $180 \mathrm{~min}$, which indicates that the adsorption process is still proceeding with a relatively low speed. Finally, the transport and transfer of cadmium ions attain equilibrium value until $120 \mathrm{~min}$. The adsorption of cadmium ions on the MH-SEP is more obvious at the initial stage, which may be attributed to the mass transfer driving force obtained from the initial concentration difference of heavy metal ions between the solid-liquid two phases and a large number of active adsorption sites on the surface of sepiolite mineral nanofibers. ${ }^{37,38}$

Fig. 7 shows the effect of the initial $\mathrm{pH}$ on the adsorption of cadmium ions by $\mathrm{MH}-\mathrm{SEP}$ at a reaction time of $120 \mathrm{~min}$. The $\mathrm{pH}$ value of solution affects the adsorption capacity of the adsorbent to the heavy metal ions, and different initial $\mathrm{pH}$ values have been reported to have significant impact on the adsorption of cadmium ions by the various adsorbents. ${ }^{39-41}$ As shown in Fig. 7, the adsorption efficiency of cadmium ions onto MH-SEP is increased from $29.8 \%$ to $68.6 \%$ when the $\mathrm{pH}$ value is increased from 2.0 to 5.0. The adsorption efficiency starts to decrease with a further increase in the $\mathrm{pH}$, and the maximum adsorption efficiency occurs at a $\mathrm{pH}$ of 5.0. The adsorption efficiency is relatively low at a $\mathrm{pH}$ value lower than 5 , which may be due to the fact that the concentration and activity of $\mathrm{H}^{+}$ions 

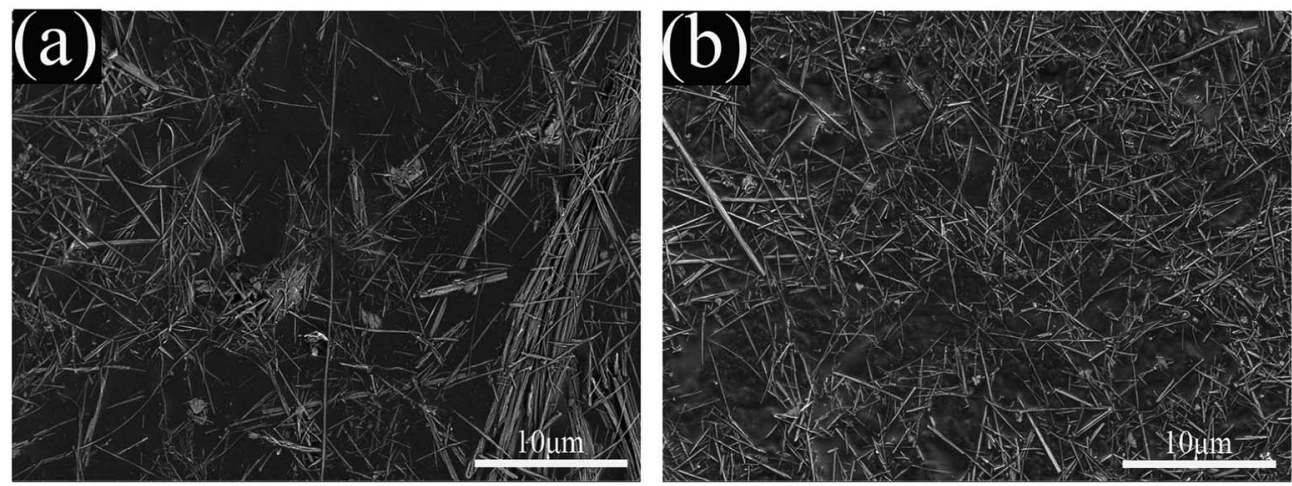

Fig. 3 SEM images of (a) A-SEP and (b) MH-SEP.

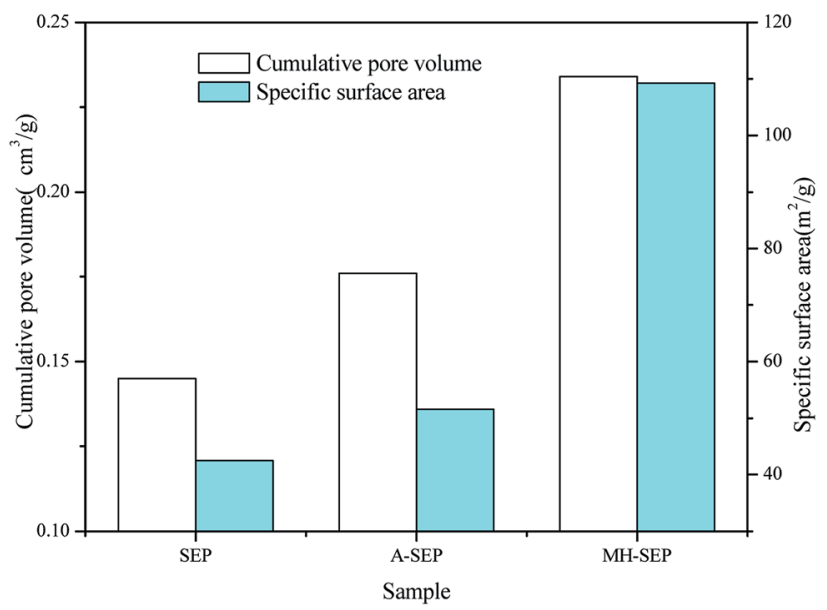

Fig. 4 Cumulative pore volume and specific surface area of the sepiolite samples.

are high, and more surface active sites of the MH-SEP could be occupied by the adsorbed $\mathrm{H}^{+}$ions, resulting in the adsorption competition with the cadmium ions. ${ }^{42,43}$ Meanwhile, the

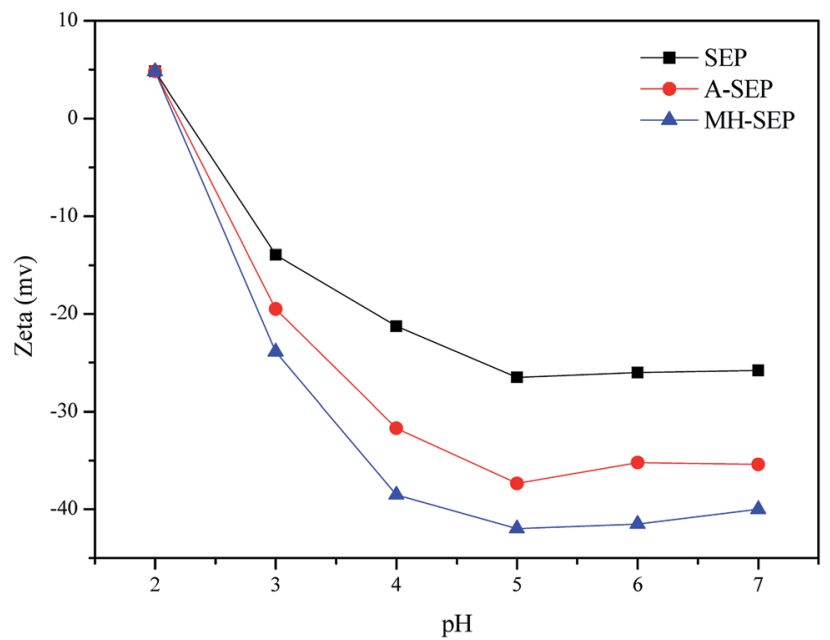

Fig. 5 Zeta potential of the various sepiolite samples at different $\mathrm{pH}$ values.

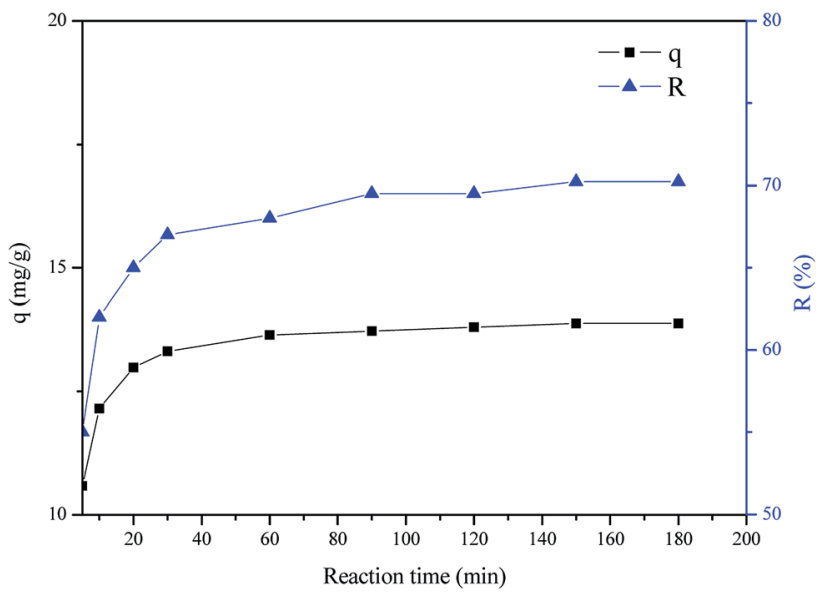

Fig. 6 Effects of the reaction time on the $\mathrm{Cd}^{2+}$ adsorption on the $\mathrm{MH}-$ SEP.

transport and transfer rate of heavy metal ions on the surface of sepiolite mineral nanofibers could be affected by the electrostatic effect between the $\mathrm{H}^{+}$and cadmium ions. As the $\mathrm{pH}$ of the solution increases, more active groups on the surface of the sepiolite fibers are exposed, which greatly promotes the contact

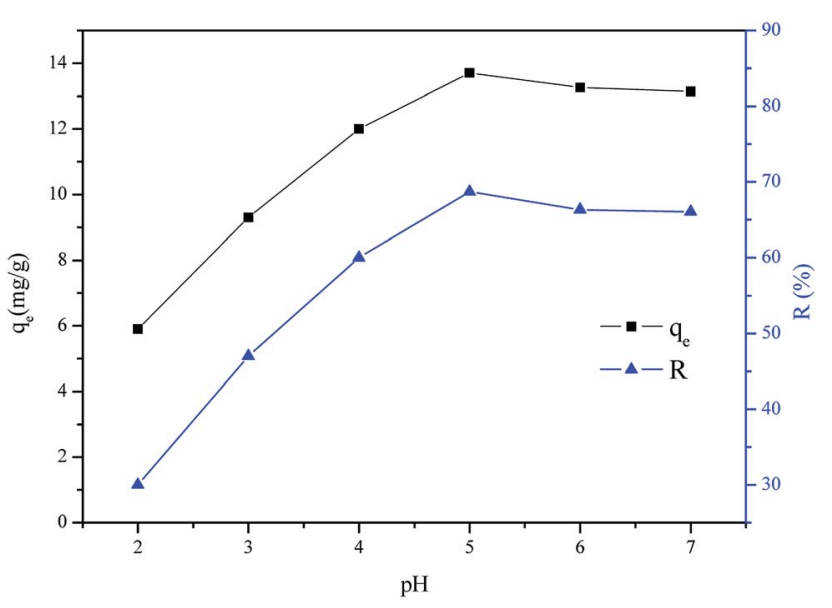

Fig. 7 Effects of initial $\mathrm{pH}$ value on $\mathrm{Cd}^{2+}$ adsorption by MH-SEP. 

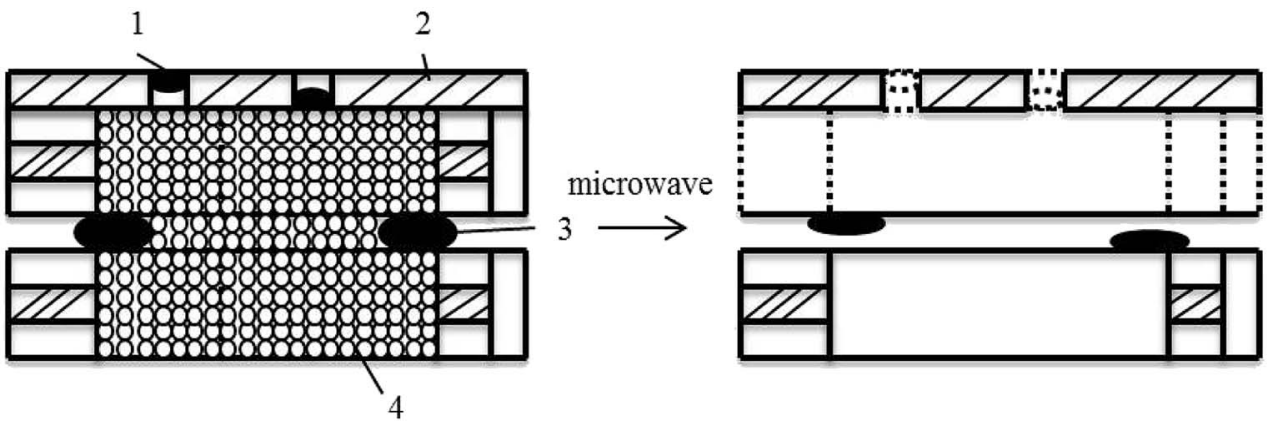

Fig. 8 Schematic diagram of the sepiolite fibers structure controlled by microwave-hydrogen peroxide ((1): channel inclusion; (2): wall of channel in sepiolite fibers; (3): fibrous bond; (4): hydrogen peroxide).

and coverage of cadmium cations onto the surface of the sepiolite mineral nanofibers. Therefore, 5 is considered to be the optimal $\mathrm{pH}$ value.

Based on the analysis above, a microwave-hydrogen peroxide method is utilized to prepare sepiolite mineral nanofibers, which could address the problem of the pores and fibers due to the defects of crystal structure and the natural heterogeneity of the sepiolite. Sepiolite has zeolite water channels and pores throughout the structure as well as a large specific surface area, and as a result, lots of water or polar substances can be adsorbed. As a polar molecule hydrogen peroxide can be adsorbed between the sepiolite fiber channels and within the fiber pores. According to the theory of surface adsorption and capillary condensation, the adsorption of hydrogen peroxide molecules by sepiolite can be concluded as the adsorption of multimolecular layers inside macropores and the capillary condensation of mesopores and micropores. At the same time, hydrogen peroxide molecules can be mainly adsorbed on the inner surface of the sepiolite mineral nanofibers. The highfrequency oscillations and the collision of the hydrogen peroxide molecules will generate much friction heat under the penetration of high-frequency electromagnetic microwaves, which results in temperature rising and eventually decomposing to produce a large amount of oxygen. ${ }^{44,45}$ The oxygen molecules move rapidly in the pores and the fiber bundle gaps, and the microstructure of sepiolite fibers could be regulated when the pressure reaches a certain level (Fig. 8). On the one hand, sufficient pressure generated by the above collisions could accelerate the splitting of the pore walls of the crystal fibers and reduces the adhesion between the fiber bundles, which could promote the dissociation of the fibers and improve the dispersion of the fibers. On the other hand, the contents of axial and lateral channels can be removed via the oxygen as mentioned above, and the closed channels can be opened through the high-speed compression diffusion movement, leading to the increase of pore volume and specific surface area of the sepiolite. The absolute value of zeta potential of the $\mathrm{MH}-$ SEP is the largest, and the most negative charge should be present on the surface of the MH-SEP at the same $\mathrm{pH}$ value, which could be helpful for the electrostatic adsorption effect on heavy metal cations. Taking cadmium ions as an example, sepiolite mineral nanofibers exhibited a good adsorption performance to cadmium ions under the optimal conditions, and the adsorption efficiency was up to $68.6 \%$.

\section{Conclusions}

In this study, we have demonstrated that the sepiolite mineral nanofibers synthesized by the microwave-hydrogen peroxide method have a low degree of aggregation. We found that this method plays an important role in the structure regulating, including the dissociation of sepiolite mineral nanofibers and the removal of the pore inclusions. The specific surface area and cumulative pore volume were $109.21 \mathrm{~m}^{2} \mathrm{~g}^{-1}$ and $0.234 \mathrm{~cm}^{3} \mathrm{~g}^{-1}$ under the optimum microwave power of $400 \mathrm{~W}$, respectively, while the zeta potential of the sepiolite mineral nanofibers reached the maximum value at $\mathrm{pH}$ 5.0. The sepiolite mineral nanofibers exhibited the best adsorption performance to cadmium ions under the conditions of a reaction time of $120 \mathrm{~min}$ and an initial $\mathrm{pH}$ of 5.0, and the adsorption efficiency was up to $68.6 \%$. The results indicate that the sepiolite mineral nanofibers as-fabricated with low price could effectively remove the heavy metal ions from aqueous solutions, which could be widely used in the purification of industrial wastewater.

\section{Conflicts of interest}

There are no conflicts to declare.

\section{Acknowledgements}

This work was financially supported by National Key R\&D Program of China (No. 2017YFB0310802), National Natural Science Foundation of China (No. 51874115), Introduced Overseas Scholars Program of Hebei Province, China (No. C201808), and Excellent Young Scientist Foundation of Hebei Province, China (No. E2018202241).

\section{References}

1 S. Deng, P. Wang, G. Zhang and Y. Dou, J. Hazard. Mater., 2016, 307, 64-72.

2 R. Karthik and S. Meenakshi, Chem. Eng. J., 2015, 263, 168177. 
3 Y. Snoussi, M. Abderrabba and A. Sayari, J. Taiwan Inst. Chem. Eng., 2016, 66, 372-378.

4 G. Chen, K. J. Shah, L. Shi and P.-C. Chiang, Appl. Surf. Sci., 2017, 409, 296-305.

5 K. Chen, J. He, Y. Li, X. Cai, K. Zhang, T. Liu, Y. Hu, D. Lin, L. Kong and J. Liu, J. Colloid Interface Sci., 2017, 494, 307316.

6 X. Wang, K. Huang, Y. Chen, J. Liu, S. Chen, J. Cao, S. Mei, Y. Zhou and T. Jing, J. Hazard. Mater., 2018, 350, 46-54.

7 C. Xu, Z. Yu, K. Yuan, X. Jin, S. Shi, X. Wang, L. Zhu, G. Zhang, D. Xu and H. Jiang, Ceram. Int., 2019, 45, 37433753.

8 S. Coruh, F. Geyikci and S. Elevli, Clays Clay Miner., 2011, 59, 617-625.

9 F. Wang, Z. Xie, J. Liang, B. Fang, Y. Piao, M. Hao and Z. Wang, Environ. Sci. Technol., 2019, 53, 6989-6996.

10 Y. C. Chemeda, G. E. Christidis, N. M. T. Khan, E. Koutsopoulou, V. Hatzistamou and V. C. Kelessidis, Appl. Clay Sci., 2014, 90, 165-174.

11 Q. Tang, F. Wang, X. Liu, M. Tang, Z. Zeng, X. Guan, J. Wang and X. Mu, Appl. Clay Sci., 2016, 132, 175-181.

12 H. Yin, Y. Yun, Y. Zhang and C. Fan, J. Hazard. Mater., 2011, 198, 362-369.

13 F. Wang, P. Gao, J. Liang, T. Zhang, H. Zhang, Y. Ding, T. Xu and B. Fang, Ceram. Int., 2019, 45, 24923-24926.

14 V. Marjanović, S. Lazarević, I. Janković-Častvan, B. Jokić, D. Janaćković and R. Petrović, Appl. Clay Sci., 2013, 80, 202-210.

15 S. Hojati and A. Landi, Desalin. Water Treat., 2015, 53, 21172124.

16 Q. Zhou, J. Huang, X. Zhang and Y. Gao, Appl. Clay Sci., 2018, 166, 307-317.

17 D. L. Guerra, A. C. Batista, P. C. Correa da Costa, R. R. Viana and C. Airoldi, J. Colloid Interface Sci., 2010, 346, 178-187.

18 C. Beauger, G. Laine, A. Burr, A. Taguet and B. Otazaghine, J. Membr. Sci., 2015, 495, 392-403.

19 F. Zhou, C. Yan, Y. Zhang, J. Tan, H. Wang, S. Zhou and S. Pu, Appl. Clay Sci., 2016, 124, 119-126.

20 Y. Zhang, L. Wang, F. Wang, J. Liang, S. Ran and J. Sun, Appl. Clay Sci., 2017, 143, 205-211.

21 J. Zhang, Z. Yan, J. Ouyang, H. Yang and D. Chen, Appl. Clay Sci., 2018, 157, 76-85.

22 J. Liang, F. Wang, Q. Tang and C. Ren, CN Pat., ZL200910070297.8, 2012.

23 L. Ge, Y. Zhang, Z. Wang, J. Zhou and K. Cen, Energy Convers. Manage., 2013, 71, 84-91.
24 B. K. Sahoo, S. De and B. C. Meikap, Fuel Process. Technol., 2011, 92, 1920-1928.

25 H. Su, C. Chen, Y. Chen, D. Yao, H. Chen, Y. Chang and T. R. Yew, Carbon, 2010, 48, 805-812.

26 Y. Zhu, S. Murali, M. D. Stoller, A. Velamakanni, R. D. Piner and R. S. Ruoff, Carbon, 2010, 48, 2118-2122.

27 F. Franco, M. Pozo, J. A. Cecilia, M. Benitez-Guerrero, E. Pozo and J. A. Martin Rubi, Appl. Clay Sci., 2014, 102, 15-27.

28 H. Li, K. Shin and G. Henkelman, J. Chem. Phys., 2018, 149, 174705.

29 H. Li and G. Henkelmane, J. Phys. Chem. C, 2017, 121, 2750427510.

30 H. Li, L. Luo, P. Kunal, C. S. Bonifacio, Z. Duan, J. C. Yang, S. M. Humphrey, R. M. Crooks and G. Henkelman, J. Phys. Chem. C, 2018, 122, 2712-2716.

31 A. Obut, I. Girgin and A. Yörükoğlu, Clays Clay Miner., 2003, 51, 452-456.

32 C. Marcos and I. Rodriguez, Appl. Clay Sci., 2014, 87, 219227.

33 W. Gan, X. Shang, X.-H. Li, J. Zhang and X. Fu, Colloids Surf., A, 2019, 561, 218-225.

34 E. Sabah, U. Mart, M. Cinar and M. S. Celik, Sep. Sci. Technol., 2007, 42, 2275-2288.

35 M. Alkan, G. Tekin and H. Namli, Microporous Mesoporous Mater., 2005, 84, 75-83.

36 S. Dikmen, G. Yilmaz, E. Yorukogullari and E. Korkmaz, Can. J. Chem. Eng., 2012, 90, 785-792.

37 Z. Zarghami, A. Akbari, A. M. Latifi and M. A. Amani, Bioresour. Technol., 2016, 205, 230-238.

38 A. A. Taha, M. A. Shreada, A. M. Ahmed and H. F. Heiba, J. Environ. Chem. Eng., 2016, 4, 1166-1180.

39 C. M. Park, J. Han, K. H. Chu, Y. A. J. Al-Hamadani, N. Her, J. Heo and Y. Yoon, J. Ind. Eng. Chem., 2017, 48, 186-193.

40 A. Kara, N. Tekin, A. Alan and A. Şafakl,, J. Environ. Chem. Eng., 2016, 4, 1642-1652.

41 E. S. Dragan, D. F. A. Loghin and A. I. Cocarta, J. Environ. Chem. Eng., 2014, 6, 16577-16592.

42 C. Fan, K. Li, J. Li, D. Ying, Y. Wang and J. Jia, J. Hazard. Mater., 2017, 326, 211-220.

43 P. Tan, J. Sun, Y. Hu, Z. Fang, Q. Bi, Y. Chen and J. Cheng, J. Hazard. Mater., 2015, 297, 251-260.

44 V. Polshettiwar and R. S. Varma, Chem. Soc. Rev., 2008, 37, 1546-1557.

45 V. A. Muromtsev, N. M. Zolotukhina and A. K. Mamina, Inorg. Mater., 1990, 26, 868-871. 